\title{
Looking for the Elusive: Mining the VLA Archives for Radio Transients
}

\author{
Destry R. Saul and Geoffrey C. Bower \\ Astronomy Department and Radio Astronomy Laboratory \\ University of California - Berkeley \\ 601 Campbell Hall \\ Berkeley, California 94720 USA
}

Received: June 7, $2006 \quad$ Accepted: July 24, 2006

\begin{abstract}
Detecting extragalactic radio transients will provide valuable information on several astronomical phenomena. Orphan gamma-ray burst afterglows (OGRBAs) and radio supernovae detection will be important for the theories of stellar collapse while the detection of tidal flares and variable active galactic nuclei (AGN) will provide details on massive black holes and galactic centers. In this paper we present the results of a survey we conducted using archived observations from the Very Large Array (VLA). We did not detect any transients in this set of data and from this we computed an upper limit on the rate of radio transients above $400 \mu$ Jansky at 0.23 transients per square degree per year. This rules out one of several theories on the rates of OGRBAs.
\end{abstract}

\section{INTRODUCTION}

Transient radio sources are objects that did not previously radiate detectably in the radio spectrum, but then begin to emit for a period of time, and then fade away. Of the several classes of predicted extragalactic radio transients, only radio supernova explosions and gamma-ray burst afterglows have been confirmed. We will discuss the different theories behind extragalactic radio transients in Section la. Detection of extragalactic radio transients will provide valuable information on the evolution of stars, black holes, and galactic centers. With this motivation, we have begun to search through the VLA archive for radio transients. The survey that we present here used data taken with the VLA from 1982 to 1992 . Our survey was superior to previous surveys due to the combined sensitivity, area, and duration of the survey. There were several difficulties with the specific field of view of our survey, and we will discuss these problems in Section II. We did not detect any viable transient candidates in our survey, and using these results we calculated an upper limit to detectable transients of 0.23 per square degree per year above $400 \mu \mathrm{Jy}$. This value agrees with some models and disagrees with others. We will discuss our results in Section III.

\section{a. A Brief Introduction to Extragalactic Radio Transient Theories}

Current theories indicate that extragalactic transient radio sources should be detectable with the VLA. Figure 1 plots the theorized rates of different types of transients. Here we provide a brief introduction to the theories of OGRBAs, tidal flares, and radio supernovae. Gamma-ray bursts are short flashes of gamma-rays that are detected roughly once a day from random directions. The origins of gammaray bursts are unknown, though there are several theories for their production. Gamma-ray bursts are separated into two categories of 'fast-hard' and 'slow-soft' by

\footnotetext{
* In astrophysics, a Jansky is a unit of measurement of flux density, in units of watt $\cdot$ meter $^{-2} \cdot$ hertz $^{-1} ; 1$ Jansky is $10^{-26} \mathrm{~W} \cdot \mathrm{m}^{-2} \cdot \mathrm{Hz}^{-1}$ and is abbreviated Jy. A microJansky is $10^{-6} \mathrm{Jy}$.
} 
the duration of the burst and the quality of

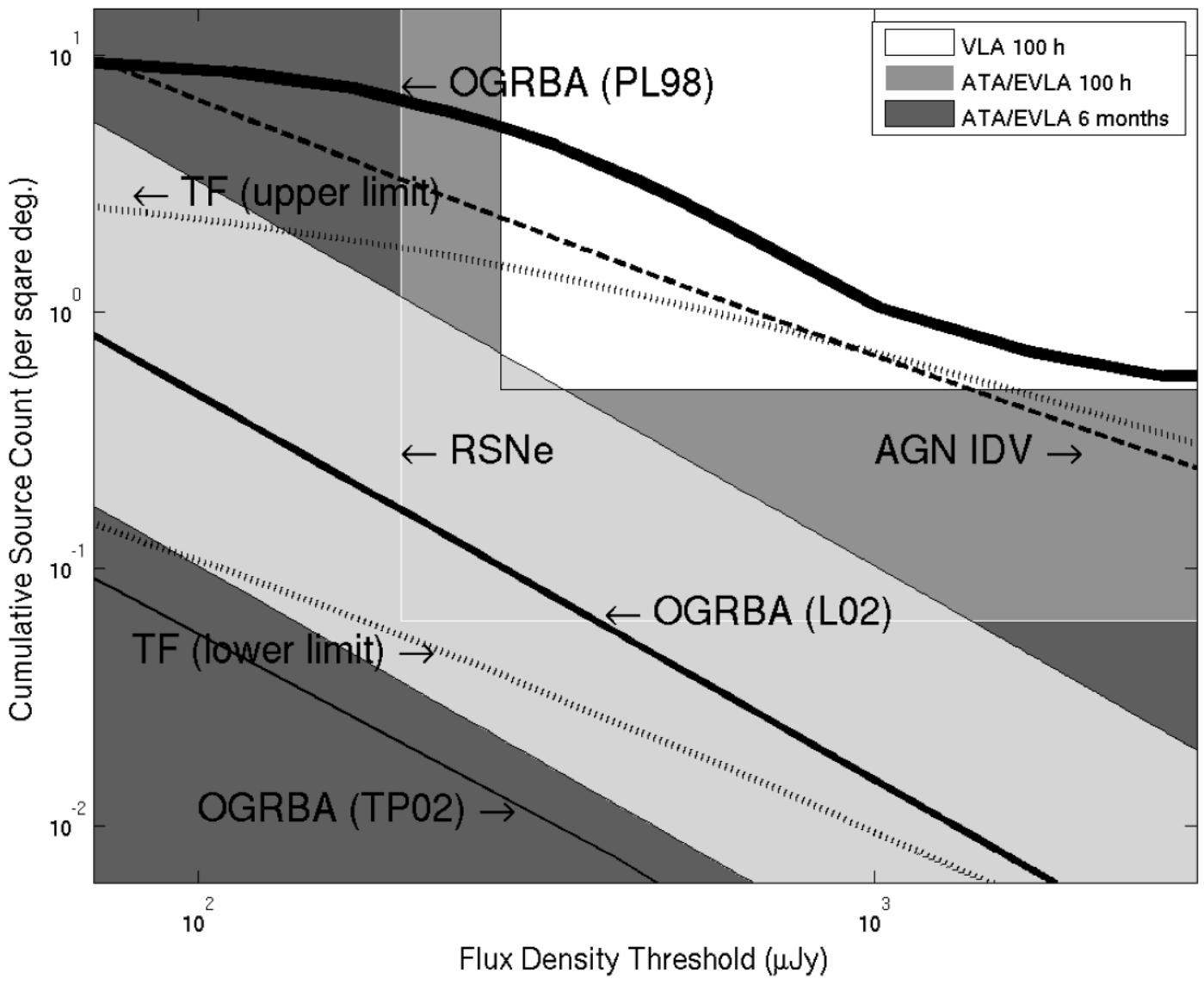

Figure 1. A plot of the different theories for radio transients. There are tidal flares (TF), radio supernova (RSNe), active galactic nuclei with intra-day variability (AGN IDV), and three models for OGRBAs: PL98 (Perna \& Loeb [9] 1998), L02 (Levinson et al. [8] 2002), and TP02 (Tolani \& Panaitescu [10] 2002). The different shaded regions represent the parameter spaces that observations with different arrays over different time durations would probe. The unshaded region is similar to the parameter space probed by our survey. Notice that the curve for OGRBA PL98 is well inside our survey. (Prepared by G. Bower)

the spectra. One theory for their cause is the collapse of a massive star into a black hole [1]. The rate of gamma-ray bursts provides information on the number and lifetime of massive stars. It would therefore be useful to have an accurate number for the rate of gamma-ray bursts. Calculating this rate has been complicated by theories that predict that gamma-ray bursts are produced in a tight beam emanating from the collapsing star's poles $[1,2]$. Thus we can only detect gamma-ray bursts produced by stars whose axis is along our line of sight. Identifying gamma-ray bursts that are not pointed directly at us involves the detection of an orphan gamma-ray burst afterglow.
Following the detection of a gammaray bursts, there have been detections of afterglows in the $\mathrm{x}$-ray through radio spectrum in the same location [3-5]. It is thought that these afterglows are caused by the deceleration of the ultra-relativistic jets emanating from the poles of the collapsing star and the interaction of these jets with the surrounding gas. This afterglow emission should be isotropic and thus detectable when the jets are not pointed toward us. This detection of an afterglow without the detection of the gamma-ray burst is an orphan gamma-ray burst afterglow. So far there have been no detections of OGRBAs in any wavelength even though there have 
been several attempts [6-8]. Searching for OGRBAs in the radio spectrum should give the best estimates on the rate of OGRBAs because the radio emission is not affected by extinction that would obscure other frequencies. The current models of OGRBAs differ significantly from each other and make it difficult to calculate the expected rate of detections. Based on one model [9], our survey should have seen several OGRBAs, but another model predicted that we would need ten times our observed area to make a detection [10]. We will present this data in Section III.

Radio transients could also be produced by the central regions of other galaxies. It has been established that there are massive black holes at the centers of most galaxies, and that some of these are 'active'. This means that there are huge amounts of energy being emitted by the central region of the galaxy. These are called active galactic nuclei (AGN). Some AGN have been shown to vary in intensity on timescales less than a day [11]. Previous surveys for AGN variability have been sensitive down to around $100 \mathrm{mJy}$. Our survey is sensitive to $400 \mu \mathrm{Jy}$ so it is possible that we could observe variability on a much more sensitive scale.

While some galactic nuclei are active, many are not. This does not mean that galaxies lacking AGN do not have massive black holes at their centers. An inactive galactic nucleus indicates that the massive black hole is not currently accreting material. It is possible that material could fall into these 'quiet' black holes and produce a bright flare of energy. This could happen when a star passes too close to the massive black hole and is torn apart by tidal forces [12]. After the star has been disrupted, it falls into the black hole and emits brightly. This emission is called a tidal flare. Tidal flares have never been detected in the radio spectrum, and there are only a few $x$-ray candidates $[13,14]$.

Of the different theories predicting extragalactic radio transients, radio supernovae are the only phenomena that have been observationally confirmed. The cause of radio emission from some supernovae is thought to be produced by the interaction of the shock produced by the supernova explosion with the gas surrounding the exploding star [15]. This gas is ejected from the star before the explosion in a process that is still hard to model. A better understanding of radio supernovae would be useful in understanding the last stages of a star's life. There have been roughly two dozen detections of radio supernovae and more would be helpful in refining the theories involved. New detections of radio supernovae could also lead to better identification of the exploding star's presupernova properties and it is also possible that radio supernovae could be used to find the distances to the galaxies where they occur [16].

Besides the types of radio transients discussed above, there is always the possibility that we will discover a previously undetected type of radio source. By using more sensitive observations of greater duration than in previous surveys we are investigating regions of parameter space that have not been observed in the past. The detection of a new type of radio source would be a very exciting discovery.

\section{OBSERVATIONS}

To observe transient radio sources, observations need to be made either over a large area of the sky, or over a long period of time. Since extragalactic radio transients should occur isotropically, the location of the observation should not affect the rate of transient detection. Because we have not had dedicated telescope time for our search, we have used data taken by other astronomers for other purposes. The data we used for this survey was taken with the VLA from 1982 to 1992 . The VLA is a 27 dish interferometer located in Socorro, New Mexico. Each dish in the array has a diameter of 25 meters. The VLA is currently the best instrument for conducting blind surveys because of the high sensitivity and the large field of view. The dishes in the array can be moved to four different configurations. The largest configuration has a maximum baseline of 36 kilometers and the smallest configuration fits all the dishes within one kilometer. The larger two configurations provide higher resolution, but also limit the field of view. We did not use the observations taken in these outer configurations because the images were significantly smaller than the images 


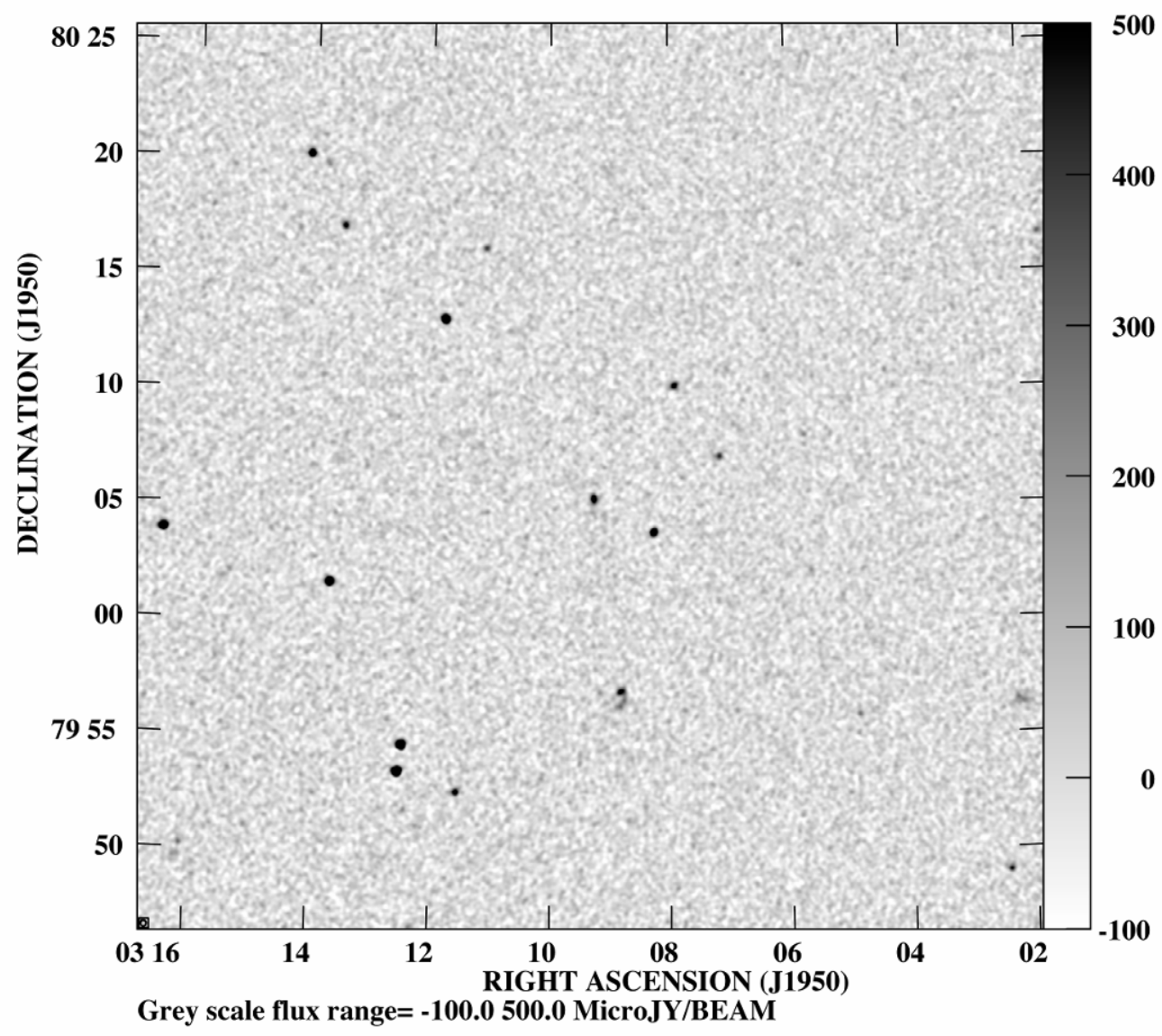

Figure 2. An image of the full field of view of our observations-notice the two bright sources in the lower left quadrant.

produced in the tighter two configurations. This left us with 16 observations from December 1982 to September 1992. Since our data was taken in the $\mathrm{L}$ Band $(1.4 \mathrm{GHz})$, each image covered 0.43 square degrees. We reduced the data using a software package developed by the National Radio Astronomy Observatory (NRAO) called the Astronomical Image Processing System (AIPS). AIPS is the standard program for reducing and analyzing VLA data. To reduce the data into a usable image we performed self-calibration on the data using the AIPS 'task' CALIB. Some of the observations had data that was corrupted for some baselines, so we removed, or 'flagged' these baselines. Once the data was appropriately flagged and calibrated, we imaged the data using the AIPS task IMAGR. After reduction, we used a combined image of all of our data to identify the constant sources in our field. It was important to establish these sources since some low level sources appeared in only one or two images. This does not make these single detections transients because they are present in the other images, just hidden in the noise. To identify sources we used an AIPS 'task' called Search And Destroy (SAD) that searched for regions of the image above given flux thresholds, fit Gaussians to these regions, and subtracted those Gaussians. SAD does this process iteratively, detecting weaker sources. We then compared the detections from each observation to the combined image. Our data was not ideal for a blind survey because it is a complicated field. Figure 2 illustrates that there are many sources in the field and several very bright sources. The two bright sources in the lower left corner were troublesome because they introduced a high noise level in that quadrant of the image. Because of this we ignored 

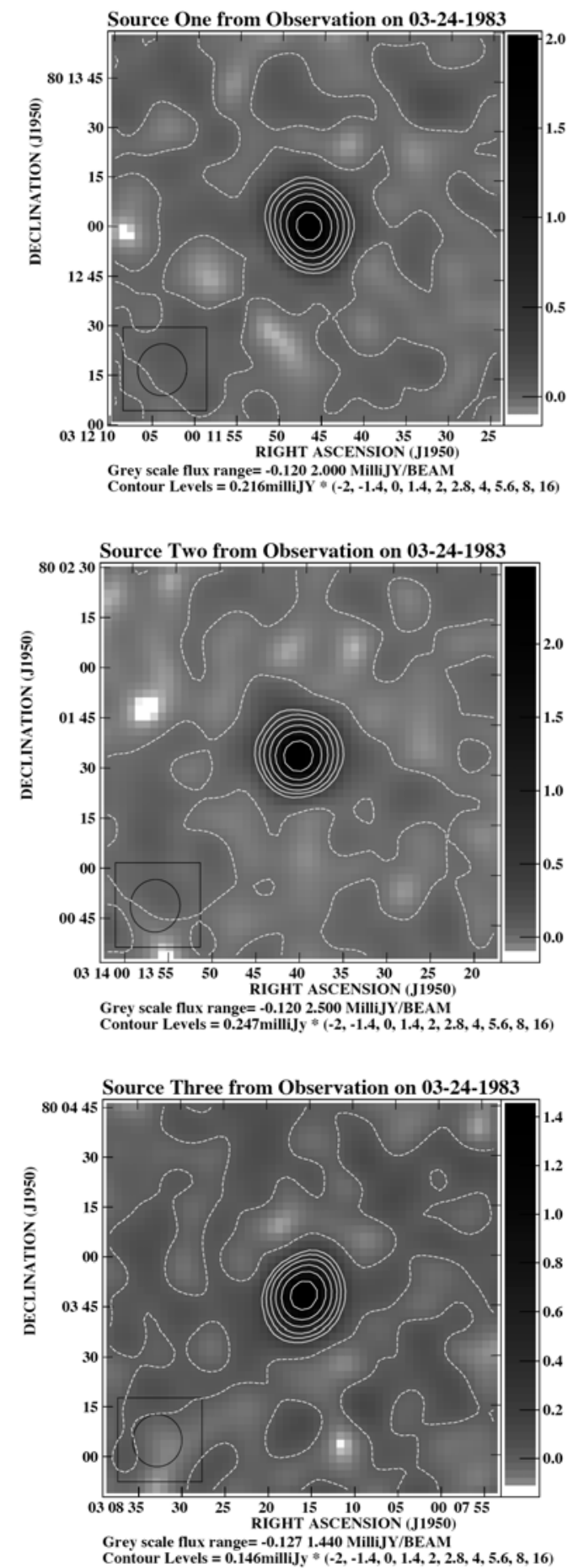
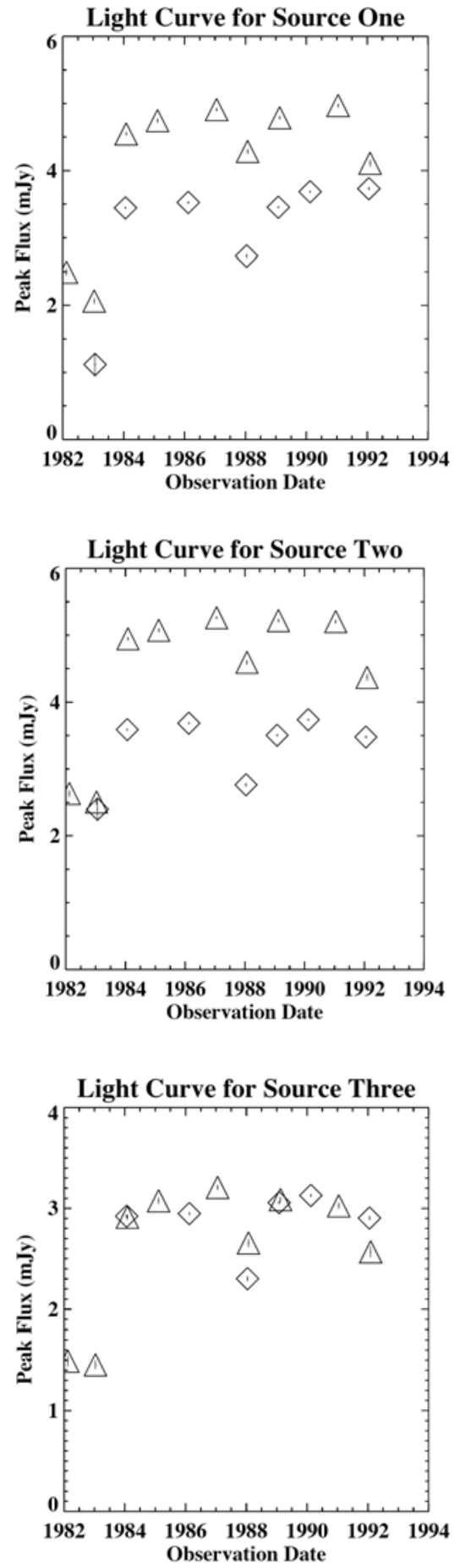

Figure 3. Three sources from our survey with corresponding light curves. In the light curves, the triangles represent observations in the $\mathrm{D}$, or most compact configuration, and the diamonds represent observations in the $\mathrm{C}$ configuration. The variation in the flux is not true variability, but a product of the different configurations of the VLA.

detections that were above the overall noise level, but near those bright sources. After analyzing the 16 observations we had a list of seven possible transients. All seven of these candidates happened to be in regions of high noise, or were just at the flux 
threshold we set for believability. It was not conclusive that any of these candidates were transient radio sources.

\section{DISCUSSION}

Our 16 observations covered a total of ten years and 0.43 square degrees. To compute the upper limit on the rate of transients we look at the limit where the length of time that the transient would be detected is larger than the spacing between our observations and the limit where the transient is detectable for a time less than the duration of our observations. Our survey was much more sensitive to the first case where the transient is detectable for at least several months. This is because our observations were on the order of twelve hours in duration, but on average separated by more than half a year. OGRBAs, tidal flares, and radio supernovae should all have detection durations on the order of months. In this limit we can compute the rate using

$$
\text { Rate }=\frac{N_{\mathrm{det}}}{\left(A_{0} \times\left(T_{2}-T_{1}\right)\right)},
$$

where $N_{\text {det }}$ is the number of detections, $A_{0}$ is the area per observation, $T_{1}$ is the date of the first observation, and $T_{2}$ is the date of the last observation. Setting $N_{\text {det }}$ equal to one we can compute an upper limit on these types of transients of 0.23 radio transients per square degree per year above the threshold of our observations. The sensitivity of our observations varied from near 100 to $400 \mu \mathrm{Jy}$. So we set our upper limit on the rate using $400 \mu \mathrm{Jy}$. For transients that are observable on time scales similar to the length of our observations (twelve hours) we can use

$$
\text { Rate }=\frac{N_{\mathrm{det}}}{\left(A_{0} \times \delta t \times N_{\text {obs }}\right)},
$$

where $\delta \mathrm{t}$ is the duration of a single observation in hours, and $\mathrm{N}_{\text {obs }}$ is the number of observations. Using this equation we can compute an upper limit of 0.012 radio transients with characteristic timescales less than 12 hours per square degree, per hour, above $400 \mu \mathrm{Jy}$. This translates to an upper limit of 106 of these radio transients per square degree per year. This limit is so high because this survey is not sensitive to short timescale transients. Figure 1 was prepared for a theoretical survey consisting of two observations separated by one year, so the computed rates are per year. Figure 1 also illustrates what theories we tested with our survey of about 100 hours. As you can see, our survey was not sensitive enough, or had great enough area, to test all of the transient predictions, though we did probe some theories. Our observations do rule out the theory of OGRBAs by Perna \& Loeb [9] since our upper limit is below the rate predicted by their calculations. But lack of detections in this survey is not that surprising. We should have detected numerous OGRBAs if Perna \& Loeb's theory was accurate, but newer predictions put OGRBAs at an order of magnitude or two less frequent. Following these models, we would need to at least double the size our survey to detect an OGRBA. The upper limit on the rate of tidal flares predicts that we could have observed a tidal flare, but the theory for tidal flares still produces a broad range for the rate of tidal flare detections.

\section{CONCLUSION}

After analyzing 6.88 square degrees of data taken over ten years we were able to set an upper limit on the rate of radio transients with timescales on the order of months at 0.23 per square degree per year above $400 \mu \mathrm{Jy}$. Though it was unlikely for this survey to detect a transient radio source, we were able to refine our process of analysis and are currently looking at more data from the VLA archives. This other set of observations is larger and more sensitive than the survey we present here, providing us with a much higher probability of finding a radio transient.

\section{ACKNOWLEDGEMENTS}

This research was completed under the guidance of Geoffrey C. Bower. I would like to thank Dr. Alberto Bolatto for his contributions and Julie Comerford for her assistance in the preparation of this paper. Funding for this research was provided by the Berkeley Radio Astronomy Laboratory.

\section{REFERENCES}

1. E. Waxman, S. R. Kulkarni, and D. A. Frail. Implications of the Radio Afterglow from the Gamma- Ray Burst of 1997 
May 8. Astrophysical Journal, 497:288293, April 1998.

2. D. A. Frail, S. R. Kulkarni, R. Sari, S. G. Djorgovski, J. S. Bloom, T. J. Galama, D. E. Reichart, E. Berger, F. A. Harrison, P. A. Price, S. A. Yost, A. Diercks, R. W. Goodrich, and F. Chaffee. Beaming in Gamma-Ray Bursts: Evidence for a Standard Energy Reservoir. Astrophysical Journal, 562:L55-L58, November 2001.

3. E. Costa, F. Frontera, J. Heise, M. Feroci, J. in: 't Zand, F. Fiore, M. N. Cinti, D. dal Fiume, L. Nicastro, M. Orlandini, E. Palazzi, M. Rapisarda, G. Zavattini, R. Jager, A. Parmar, A. Owens, S. Molendi, G. Cusumano, M. C. Maccarone, S. Giarrusso, A. Coletta, L. A. Antonelli, P. Giommi, J. M. Muller, L. Piro, and R. C. Butler. Discovery of an X-ray afterglow associated with the gamma-ray burst of 28 February 1997. Nature, 387:783-785, June 1997.

4. J. van Paradijs, P. J. Groot, T. Galama, C. Kouveliotou, R. G. Strom, J. Telting, R. G. M. Rutten, G. J. Fishman, C. A. Meegan, M. Pettini, N. Tanvir, J. Bloom, H. Pedersen, H. U. Nordgaard-Nielsen, M. Linden-Vornle, J. Melnick, G. van der Steene, M. Bremer, R. Naber, J. Heise, J. in 't Zand, E. Costa, M. Feroci, L. Piro, F. Frontera, G. Zavattini, L. Nicastro, E. Palazzi, K. Bennet, L. Hanlon, and A. Parmar. Transient optical emission from the error box of the gamma-ray burst of 28 February 1997. Nature, 386:686-689, 1997.

5. D. A. Frail, S. R. Kulkarni, S. R. Nicastro, M. Feroci, and G. B. Taylor. The radio afterglow from the gamma-ray burst of 8 May 1997. Nature, 389:261263, 1997.

6. E. V. Gotthelf, T. T. Hamilton, and D. J. Helfand. The Einstein Observatory Detection of Faint X-Ray Flashes. Astrophysical Journal, 466:779-793,
August 1996.

7. A. Gal-Yam, E. O. Ofek, A. V. Filippenko, R. Chornock, and W. Li. SDSS J124602.54+011318.8: A Highly Variable Active Galactic Nucleus, Not an Orphan Gamma-Ray Burst Afterglow. PASP, 114:587-592, June 2002.

8. A. Levinson, E. O. Ofek, E. Waxman, and A. Gal-Yam. Orphan Gamma-Ray Burst Radio Afterglows: Candidates and Constraints on Beaming. Astrophysical Journal, 576:923-931, September 2002.

9. R. Perna and A. Loeb. Constraining the Beaming of Gamma-Ray Bursts with Radio Surveys. Astrophysical Journal, 509:L85-L88, December 1998.

10. T. Totani and A. Panaitescu. Orphan Afterglows of Collimated Gamma-Ray Bursts: Rate Predictions and Prospects for Detection. ApJ, 576:120-134, September 2002.

11. S. J. Wagner and A. Witzel. Intraday Variability In Quasars and BL Lac Objects. ARA\&A, 33:163-198, 1995.

12. M. J. Rees. 'Dead quasars' in nearby galaxies? Science, 247:817-823, February 1990.

13. S. Komossa. The extremes of (X-ray) variability among galaxies: Flares from stars tidally disrupted by supermassive black holes. In IAU Symposium, pages 45-48, November 2004.

14. L.-X. Li, R. Narayan, and K. Menou. The Giant X-Ray Flare of NGC 5905: Tidal Disruption of a Star, a Brown Dwarf, or a Planet? Astrophysical Journal, 576:753761, September 2002.

15. R. A. Chevalier. The radio and X-ray emission from type I supernovae. Astrophysical Journal, 259:302-310, August 1982.

16. K. W. Weiler, S. D. van Dyk, M. J. Montes, N. Panagia, and R. A. Sramek. Radio Supernovae as Distance Indicators. Astrophysical Journal, 500:51-58, June 1998. 


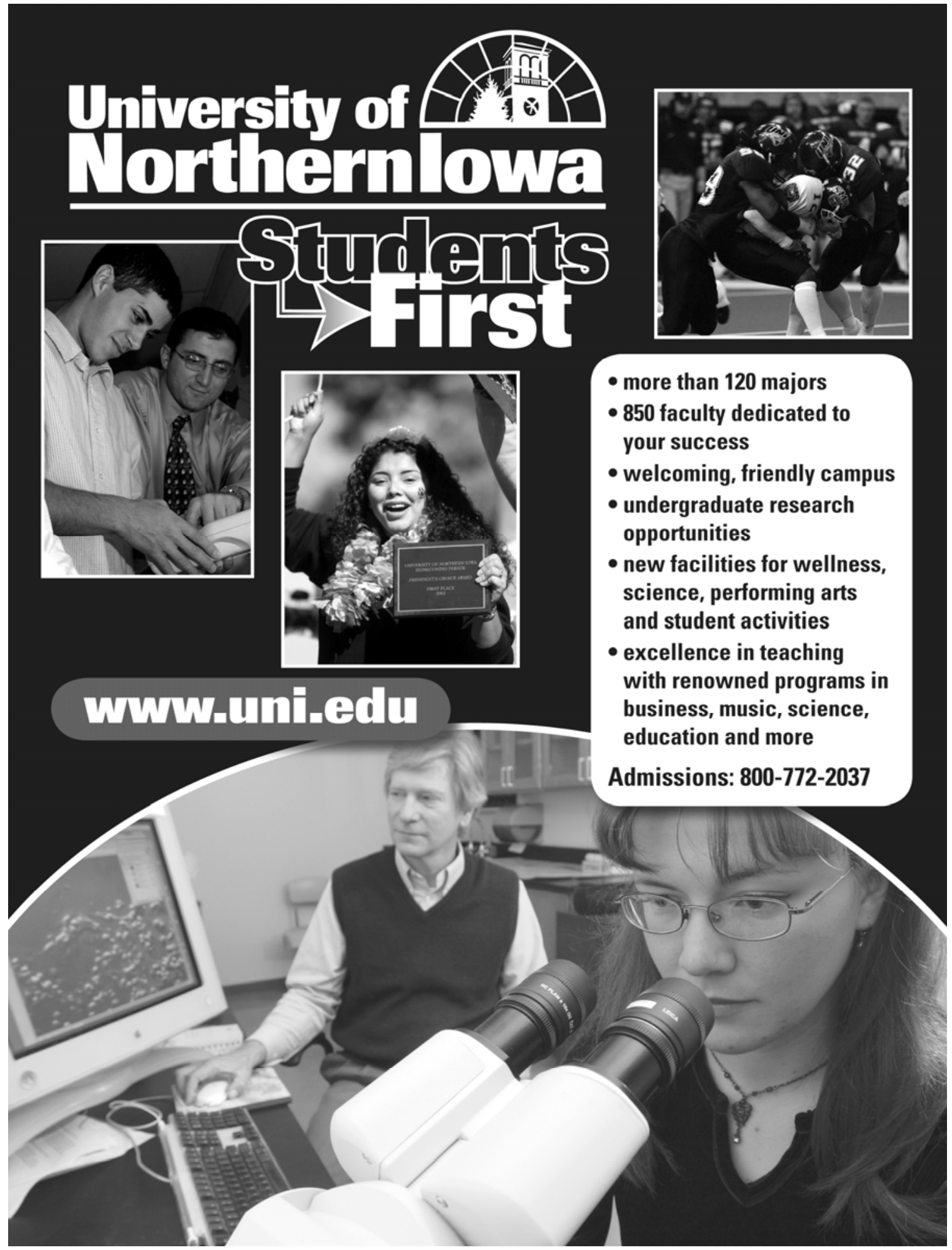

